\title{
The Importance of Recognizing Diffuse Idiopathic Skeletal Hyperostosis for Neurosurgeons: A Review
}

\author{
Masatoshi YunOKI, ${ }^{1}$ Kenta SuZUKI, ${ }^{1}$ Atsuhito UnEDA, ${ }^{1}$ Shuichi OKUBO, ${ }^{1}$ \\ Koji HIRASHITA, ${ }^{1}$ and Kimihiro YOSHINO ${ }^{1}$
}

${ }^{1}$ Department of Neurosurgery, Kagawa Rosai Hospital, Marugame, Kagawa

\begin{abstract}
Diffuse idiopathic skeletal hyperostosis (DISH) is characterized by calcification and ossification of the soft tissues, mainly ligaments and entheses. The spines of patients with DISH generally become increasingly rigid and osteoporotic, and fractures may occur after even a relatively minor traumatic event such as a ground-level fall. Moreover, the prevalence of DISH may be rapidly increasing in affluent societies. Thus, awareness of this condition is becoming more important for neurosurgeons when assessing trauma patients. For the present article, a literature review was conducted to summarize the current clinical, pathogenetic, and therapeutic knowledge of this disease. Furthermore, current treatment strategies for DISH-related spine injuries are also reviewed. Although the recommended treatment for spinal injuries in DISH patients is surgical, mainly through long-segment posterior fusion, rather than conservative options, stable fractures without any associated neurologic deficits have often been successfully managed with immobilization alone. Percutaneous instrumentation and the use of teriparatide may be useful depending on the surgical risks and patient neurological status.
\end{abstract}

Key words: spinal fracture, diffuse idiopathic skeletal hyperostosis, trauma, surgery

\section{Introduction}

Diffuse idiopathic skeletal hyperostosis (DISH) is an idiopathic form of degenerative arthritis that typically affects males over 60 years of age. ${ }^{1-6)}$ DISH is characterized by exuberant bony growth along the anterior longitudinal ligament. ${ }^{1)}$ Because the condition is usually painless and latent, DISH is often incidentally diagnosed by radiographic examination taken for a non-related reason. DISH causes ankylosis of the spinal column, which can make the spine prone to fracture after even trivial trauma. Moreover, such unstable fractures can lead to secondary neurologic deterioration in initially asymptomatic patients. ${ }^{2-6)}$ The prevalence of DISH may be rapidly increasing in affluent societies. Thus, awareness of this condition should be increased among neurosurgeons that assess trauma patients and treat spinal injuries. ${ }^{5)}$ In the present article, a review of the literature was conducted to summarize the current clinical, pathogenetic, and therapeutic knowledge of this disease. In addition, the current treatment strategies for DISH-related spine injuries are also reviewed.

Received January 13, 2016; Accepted February 20, 2016

\section{Disease Characteristics and Diagnosis of DISH}

DISH, then termed senile ankylosing hyperostosis, was first described by Forestier and Rotes-Querol more than 50 years ago. ${ }^{1}$ Later, because a similar condition was also recognized in younger patients, the name ankylosing spinal hyperostosis (ASH) was adopted.7) As the involvement of the axial skeleton, particularly the thoracic spine and the peripheral joints were recognized, researchers began using the term DISH. ${ }^{8)}$ The disease mainly targets the enthesis, an organ that is rich in collagen fibers, fibroblasts, other mesenchymal cells, fibrocartilage, and calcified matrix that penetrates the bone cortex at its attachment. Although there are no validated diagnostic criteria for DISH, three sets of criteria are often used for diagnosis. The most commonly used classification criteria were defined by Resnick and Niwayama. These criteria include the involvement of at least four contiguous vertebrae of the thoracic spine, preservation of the intervertebral disc space, and the absence of apophyseal joints and sacroiliac inflammatory changes. ${ }^{8}{ }^{8}$ The criteria defined by Julkunen et al. are nearly equivalent to those of Resnick and Niwayama, except that they include bridges connecting two vertebral bodies in at least two 
sites on the thoracic spine, which are also reported to be involved in DISH. ${ }^{9)}$ Neither of these two sets of criteria assess the peripheral manifestations of the condition. However, the criteria for DISH defined by Utsinger et al. lowered the threshold for spinal involvement to three contiguous vertebral bodies, but added the presence of peripheral enthesopathies to the diagnostic measures (Table 1). ${ }^{10)}$

The differential diagnosis of DISH requires distinguishing it from ankylosing spondylitis (AS). An AS is a relatively rare disease with a prevalence of $0.05-1.4 \%$, whereas the DISH has a prevalence ranging from $2.9 \%$ to $25 \%{ }^{5,11)}$ It is a chronic inflammatory rheumatic disease that tends to affect relatively young white males. ${ }^{5,11)}$ Patients with AS are usually symptomatic, but suffer from a myriad of associated conditions such as iritis, uveitis, or ulcerative colitis. Sacroiliac and apophyseal fusion or sclerosis, the earliest symptoms of which are back pain and stiffness, result from inflammation of the sacroiliac joint. ${ }^{11)}$ This inflammation can gradually spread to the joints between the vertebrae, causing a condition called spondylitis, as well as to other joints including the

Table 1 Diagnostic criteria for diffuse idiopathic skeletal hyperostosis

Resnick and Niwayama criteria

1. Flowing ossification along the anterolateral aspect of at least four contiguous vertebral bodies

2. Relative preservation of intervertebral disc height in the involved segment

3. Absence of apophyseal joint bony ankylosis and sacroiliac joint erosion

Julkunen criteria

Substantially the same as the Resnick and Niwayama criteria but slightly different in that bridges connecting the two vertebral bodies in at least two sites on the thoracic spine are also included

Utsinger criteria

Bridging of four contiguous vertebral bodies Definite primarily in thoracolumbar spine, minimal

DISH intervertebral disc disease, no facet joint ankylosis

Probable Bridging of two contiguous vertebral bodies DISH plus bilateral patellar tufting, heel spurring, and olecranon tufting

Two vertebrae joined in the absence of extraPossible spinal enthesophytes or symmetrical extraDISH spinal enthesophytes in the absence of spinal involvement

DISH: diffuse idiopathic skeletal hyperostosis. shoulders, hips, and knees. In severe cases, the illness can result in characteristic postural abnormalities (e.g., "Bechterew stoop") after many years. An AS is likely caused by the combination of genetic and environmental factors, most of which have not yet been identified. However, researchers have found several genes that influence the risk of developing this disorder, such as HLA-B27, ERAP1, IL1A, and IL23R. ${ }^{11)}$

\section{Epidemiology and Etiology of DISH}

The exact epidemiology of DISH has not yet been described in the medical literature, certainly because of the lack of consensus concerning an exact definition of the disease. However, a few well-designed studies have attempted to estimate its prevalence. Holton et al. examined 298 men aged older than 65 years from the general population and found the prevalence of DISH, as assessed by the criteria of Resnick and Niwayama, was $42 \% .{ }^{12)}$ Cassim et al. examined the prevalence of DISH in hospitalized black Africans over the age of 40 years. They reported that the overall prevalence of DISH was 3.9\% (3.8\% in males and $4.2 \%$ in females). There was an increase in the prevalence of DISH with increasing age from $1 \%$ in the $40-49$-year-age group to $13.6 \%$ in those over 70 years. ${ }^{13)}$ Furthermore, Weinfeld et al. analyzed data from two large American midwest metropolitan hospital populations to conclude that the prevalence of DISH in that mostly Anglo-Saxon population over 50 years of age was $25 \%$ in males and $15 \%$ in females. At 70 years of age, furthermore, the prevalence in males and females increased to $35 \%$ and $26 \%{ }^{14)}$ Based on these data, DISH is likely to be more frequent and more severe in men throughout life, and the incidence of the disease increases with age. Furthermore, a higher prevalence was reported in Caucasian populations than in Korean or black Africans. ${ }^{13,15)}$ Thus, ethnic factors are also likely important factors in the prevalence of DISH.

The etiology of DISH also remains unclear. However, several metabolic conditions (obesity, diabetes mellitus, hypertension, hyperinsulinemia, and dyslipidemia, among others), genetic factors human leukocyte antigen (HLA) and collagen type VI alpha 1 (COL6A1) genes, environmental exposures (fluoride, vitamin A/retinol), and drugs (isotretinoin, etretinate, acitretin, and other vitamin A derivatives) are reported to be associated with DISH. ${ }^{1-6)}$ In addition, two recent studies showed that patients with DISH have a higher incidence of risk factors for stroke, a higher prevalence of metabolic syndrome, and a higher risk for future coronary events. Thus, they are encouraged to take measures to reduce the risk of such complications. ${ }^{16,17)}$ 


\section{Etiology and Diagnosis of Spinal Fracture in DISH Patients}

Because DISH exposes injury sites to larger moments via the lever arms produced by stiffness of the otherwise largely ankylosed spinal column, even harmless-appearing fractures can become highly unstable and place the patient at high risk for a neurologic injury, particularly if the cervical segment is involved. ${ }^{2,4-6,18,19)}$ Previous studies have shown that these fractures most commonly occur in the cervical spine, followed by the thoracic and lumbar segments. ${ }^{5,6,19)}$ The restricted motion and overall kyphotic spinal alignment of patients with DISH increase the risk of hyperextension injuries after seemingly trivial trauma, such as falls from standing. Westerveld et al. reported in a systematic literature review that extension-type fractures occurred in 22 of 43 patients (51.2\%) with DISH, whereas rotation-type fracture occurred in 15 patients $(34.9 \%)$, compression-type fracture occurred in 6 patients $(14.0 \%)$, and flexion-type fractures in none of the DISH patients. ${ }^{5)}$ In addition, a higher incidence of missed fractures in these patients have been reported to led to the higher rates of spinal cord injury. ${ }^{2,4-6,18)}$ In DISH-related spine fractures, delayed diagnosis is reported to be particularly common in nondisplaced fractures after trivial injuries and was associated with neurologic worsening. ${ }^{19)}$ Because of the difficulty in radiographically visualizing spines affected by DISH, screening of the entire spinal column with an advanced neuroimaging modality (magnetic resonance imaging or computed tomography) has been recommended especially for patients with persistent neck or back pain. ${ }^{6)}$

In Japan, there are many facilities where trauma patients whose main injury site is the head are treated predominantly by neurosurgeons. Especially when head trauma is accompanied by an intracranial hemorrhage (ICH), patients with even mild symptoms are admitted directly to the Department of Neurosurgery to avoid delayed deterioration. ${ }^{20)}$ During the initial management of traumatic ICH, the diagnosis of spinal fracture is difficult because of an altered level of consciousness (Fig. 1). In addition, symptoms
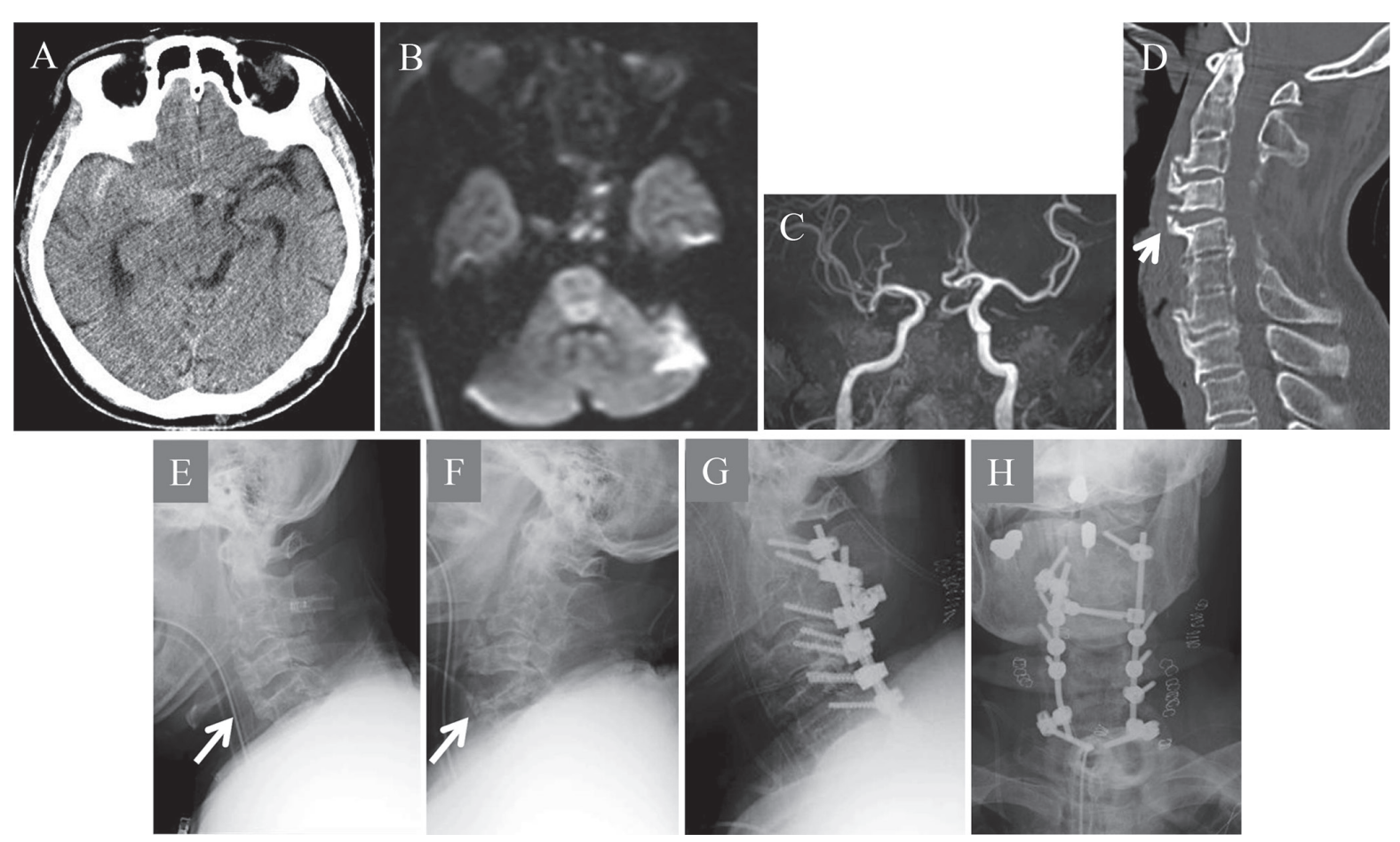

Fig. 1 A case of cervical spinal fracture with diffuse idiopathic skeletal hyperostosis (DISH) accompanying traumatic subarachnoid hemorrhage. After falling from a low height, a 79-year-old man presented with a laceration of the forehead. His consciousness level was 300 (JCS). He was admitted to the Department of Neurosurgery because head CT and MRI scans revealed traumatic subarachnoid hemorrhage (A). MRI and MR angiography detected pontine infarction and decreased blood flow in the vertebrobasilar artery (B, C). Cervical CT detected a small crack (arrow) in the anterior osteophyte of C4/5 in the setting of ankylosed spine due to DISH (D). Dynamic cervical spine XP showed an extension fracture through the C4/5 disc space (E, F). After 30 days of conservative treatment to stabilize his vital signs, he underwent posterior fixation surgery (G, H). 

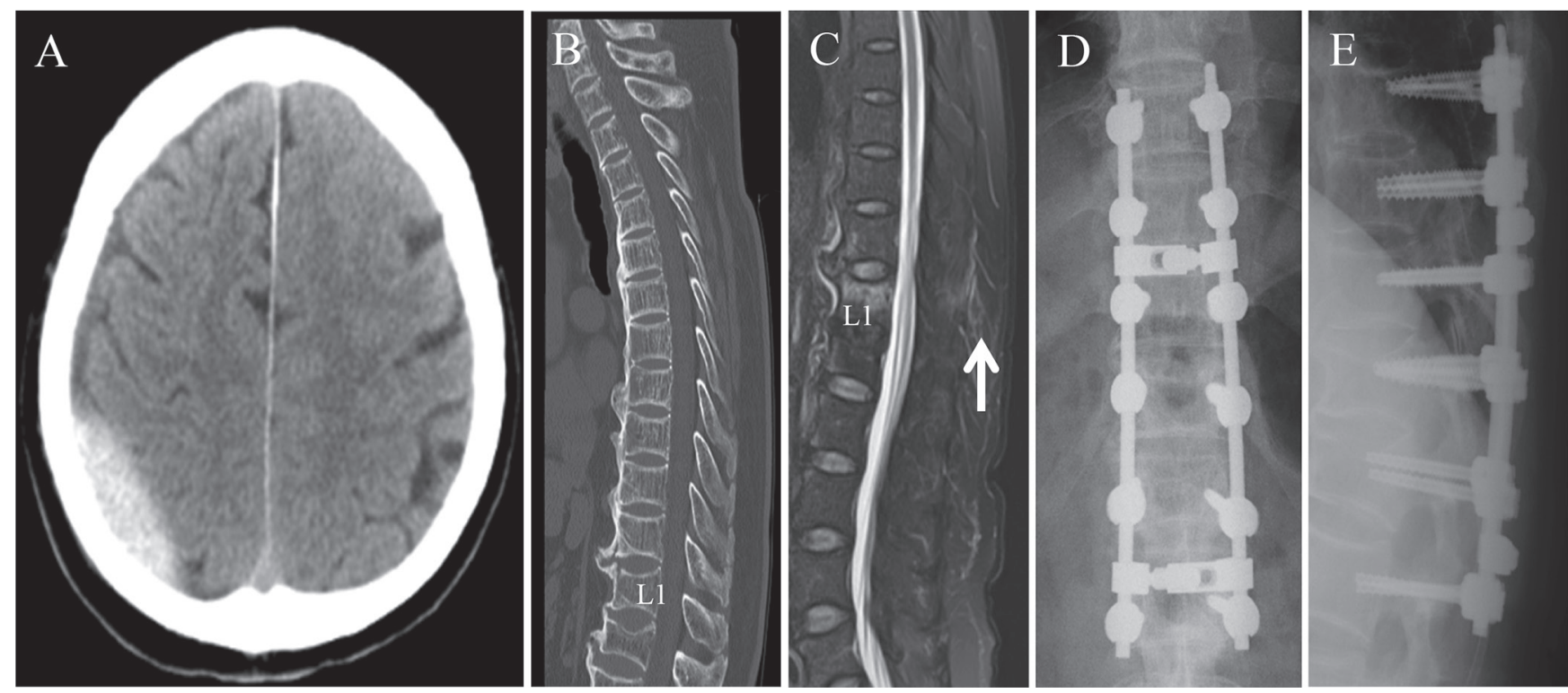

Fig. 2 A case of lumbar spinal fracture with DISH accompanying an epidural hematoma. A 75-year-old man suffered a ground-level fall and hit his occipital head. His consciousness level was alert, and no neurological deficit was identified. Because a slight right epidural hematoma was identified, he was admitted to the Department of Neurosurgery and was treated conservatively. Thoracolumbar CT was performed because he complained of persistent lumbar back pain. It revealed ankylosed spine due to DISH. Subsequent MRI was performed, and an L1 compression fracture involving the posterior element was identified. Posterior fixation surgery was performed 21 days after admission ( $G, H)$.

associated with spinal fracture in DISH patients may be minor (Fig. 2). Neurosurgeons therefore must be careful to remain aware of the possible presence of DISH in these patients.

\section{Treatment of Spinal Fractures in DISH Patients}

Treatment for ankylosed spine fractures in DISH patients may be operative or nonoperative depending on the patient age, medical comorbidities, fracture pattern, and neurologic status. Conservative treatment is usually performed by bracing and bed rest. However, surgical rather than conservative treatment is generally recommended based on the incidence of complications and the prognosis of such fractures. ${ }^{5)}$ Caron et al. reported that the mortality rate of the surgically-treated group in their study was $23 \%$, lower than the $51 \%$ mortality rate with no operative treatment. Further, the mortality rate was significantly lower in patients aged 70 years or older. ${ }^{19)}$ It should be noted that nonoperative treatments are typically chosen because of a high surgical risk and most morbidity is cause by the development of pulmonary complications. ${ }^{5,19)}$ Whang et al. reported that stable fractures without any associated neurologic deficits can often be successfully managed with immobilization alone. ${ }^{6)}$ Moreover, a recent report described the efficacy of teriparatide (TPTD) treatment for spine fractures in patients with DISH. ${ }^{21)}$ Beyond the use for fresh fractures, the successful treatment of a lumbar spine fracture with delayed union in a patient with DISH using daily administration of TPTD was also reported. ${ }^{22)}$ In cases where surgical treatment is not indicated because of poor general condition, TPTD treatment is one option.

\section{Surgical Procedure}

A variety of surgical approaches have been reported to treat fractures in patients with DISH, including anterior, posterior, and combined approaches. However the most common approach based on the published literature is posterior instrumentation. ${ }^{4-6,18,19)}$ Because of the torque associated with the lever arms in conjunction with poor, osteoporotic bone quality, posterior surgery often requires multiple points of pedicle screw fixation above and below the affected level to appropriately stabilize the spinal column and heal the fracture. ${ }^{4-6,18,19)}$ Most reported case series on surgery for fractures in DISH patients were based on traditional open procedures with pedicle screw placement and the creation of a fusion bed. ${ }^{1,9,12,13,15)}$ However, percutaneous instrumentation has become more popular for the 
treatment of thoracolumbar trauma, ${ }^{23)}$ though this approach has been criticized for the lack of data regarding the rates of pseudarthrosis and hardware failure when used for fractures in DISH patients. Recently, Nayak et al. reported that percutaneous instrumentation can be successfully used to manage spinal fractures in patients with DISH while preserving a good postoperative quality-of-life with limited disability when used on patients with good preoperative neurologic status. ${ }^{24)}$

\section{Conclusion}

Recognition of DISH is important for neurosurgeons when assessing trauma patients because this condition can make the spine prone to fracture after even trivial trauma, which frequently results in delayed diagnosis and neurologic deterioration. Although the current recommendation is for these spinal injuries to be treated surgically, mainly through long-segment posterior fusion, rather than conservatively, stable fractures without any associated neurologic deficits have often been successfully managed with immobilization alone. Percutaneous instrumentation and the use of TPTD are other options depending on the risk of surgery and patient neurological status.

\section{Conflicts of Interest Disclosure}

The authors have no personal, financial, or institutional conflicts of interest regarding any of the drugs, materials, or devices in this article. The authors who are members of the Japan Neurosurgical Society (JNS) have registered online and filled the Self-reported COI Disclosure Statement Forms through the JNS members website.

\section{References}

1) Forestier J, Rotes-Querol J: Senile ankylosing hyperostosis of the spine. Ann Rheum Dis 9: 321-330, 1950

2) Hunter T, Forster B, Dvorak M: Ankylosed spines are prone to fracture. Can Fam Physician 41: 1213-1216, 1995

3) Meyer PR Jr: Diffuse idiopathic skeletal hyperostosis in the cervical spine. Clin Orthop Relat Res 49-57, 1999

4) Paley D, Schwartz M, Cooper P, Harris WR, Levine AM: Fractures of the spine in diffuse idiopathic skeletal hyperostosis. Clin Orthop Relat Res 22-32, 1991

5) Westerveld LA, Verlaan JJ, Oner FC: Spinal fractures in patients with ankylosing spinal disorders: a systematic review of the literature on treatment, neurological status and complications. Eur Spine J 18: 145-156, 2009

6) Whang PG, Goldberg G, Lawrence JP, Hong J, Harrop JS, Anderson DG, Albert TJ, Vaccaro AR: The management of spinal injuries in patients with ankylosing spondylitis or diffuse idiopathic skeletal hyperostosis: a comparison of treatment methods and clinical outcomes. J Spinal Disord Tech 22: 77-85, 2009

7) Forestier J, Lagier R: Ankylosing hyperostosis of the spine. Clin Orthop Relat Res 74: 65-83, 1971

8) Resnick D, Niwayama G: Radiographic and pathologic features of spinal involvement in diffuse idiopathic skeletal hyperostosis (DISH). Radiology 119: 559-568, 1976

9) Julkunen H, Heinonen OP, Knekt P, Maatela J: The epidemiology of hyperostosis of the spine together with its symptoms and related mortality in a general population. Scand J Rheumatol 4: 23-27, 1975

10) Utsinger PD, Resnick D, Shapiro R: Diffuse skeletal abnormalities in Forestier disease. Arch Intern Med 136: 763-768, 1976

11) Ghasemi-Rad M, Attaya H, Lesha E, Vegh A, MalekiMiandoab T, Nosair E, Sepehrvand N, Davarian A, Rajebi H, Pakniat A, Fazeli SA, Mohammadi A: Ankylosing spondylitis: a state of the art factual backbone. World J Radiol 28: 236-252, 2015

12) Holton KF, Denard PJ, Yoo JU, Kado DM, BarrettConnor E, Marshall LM; Osteoporotic Fractures in Men (MrOS) Study Group: Diffuse idiopathic skeletal hyperostosis and its relation to back pain among older men: the MrOS Study. Semin Arthritis Rheum 41: 131-138, 2011

13) Cassim B, Mody GM, Rubin DL: The prevalence of diffuse idiopathic skeletal hyperostosis in African blacks. Br J Rheumatol 29: 131-132, 1990

14) Weinfeld RM, Olson PN, Maki DD, Griffiths HJ: The prevalence of diffuse idiopathic skeletal hyperostosis (DISH) in two large American Midwest metropolitan hospital populations. Skeletal Radiol 26: 222-225, 1997

15) Kim SK, Choi BR, Kim CG, Chung SH, Choe JY, Joo KB, Bae SC, Yoo DH, Jun JB: The prevalence of diffuse idiopathic skeletal hyperostosis in Korea. J Rheumatol 31: 2032-2035, 2004

16) Mader R, Novofestovski I, Adawi M, Lavi I: Metabolic syndrome and cardiovascular risk in patients with diffuse idiopathic skeletal hyperostosis. Semin Arthritis Rheum 38: 361-365, 2009

17) Miyazawa N, Akiyama I: Diffuse idiopathic skeletal hyperostosis associated with risk factors for stroke: a case-control study. Spine 31: E225-E229; discussion E230, 2006

18) Bransford RJ, Koller H, Caron T, Zenner J, Hitzl W, Tomasino A, Mayer M: Cervical spine trauma in diffuse idiopathic skeletal hyperostosis: injury characteristics and outcome with surgical treatment. Spine 37: 1923-1932, 2012

19) Caron T, Bransford R, Nguyen Q, Agel J, Chapman J, Bellabarba C: Spine fractures in patients with 
ankylosing spinal disorders. Spine (Phila Pa 1976) 35: E458-E464, 2010

20) Karibe H, Hayashi $T$, Hirano $T$, Kameyama M, Nakayama A, Tominaga T: Clinical characteristics and problems of traumatic brain injury in the elderly. Jpn Neurosurg 23: 965-972, 2014

21) Iida Y, Takahashi H, Yokoyama Y, Inoue Y, Suzuki D, Hasegawa K, Tsuge S, Shishikura W, Fukutake K, Takamatsu R, Nakamura K, Sekiguchi M, Wada A: Successful treatment of spine fracture for diffuse idiopathic skeletal hyperostosis with teriparatide-a report of two cases. Open J Orthop 3: 278-282, 2013

22) Matsumoto T, Ando M, Sasaki S: Effective treatment of delayed union of a lumbar vertebral fracture with daily administration of teriparatide in a patient with diffuse idiopathic skeletal hyperostosis. Eur Spine J 24: S573-S576, 2015
23) Verlaan JJ, Diekerhof CH, Buskens E, van der Tweel I, Verbout AJ, Dhert WJ, Oner FC: Surgical treatment of traumatic fractures of the thoracic and lumbar spine: a systematic review of the literature on techniques, complications, and outcome. Spine (Phila Pa 1976) 29:803-814, 2004

24) Nayak NR, Pisapia JM, Abdullah KG, Schuster JM: Minimally invasive surgery for traumatic fractures in ankylosing spinal diseases. Global Spine $J$ 5: 266-273, 2015

Address reprint requests to: Masatoshi Yunoki, MD, Department of Neurosurgery, Kagawa Rosai Hospital, 3-3-1 Joto-cho, Marugame, Kagawa, Japan. e-mail: yunomasato@yahoo.co.jp 